\title{
Sistema de recepción de información satelital basado en
}

\section{SDR}

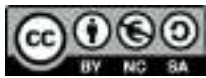

\section{System of reception of satellite information based on SDR}

Mónica Zabala. ${ }^{1}$, Gary Cabeza. ${ }^{2}$, Mayra Pacheco. ${ }^{3}$, Byron Casignia. ${ }^{4}$. \& Alejandra Oñate. ${ }^{5}$

Recibido: 27-03-2019 / Revisado: 25-04-209 /Aceptado: 19-05-2019/ Publicado: 05-06-2019

\begin{abstract}
.
DOI: https://doi.org/10.33262/cienciadigital.v3i2.6.591

The implementation of a satellite information reception system based on SDR starts from the fact of imitating the Outernet service, which is to provide basic information on meteorology, news, sports, etc, through the constellation of cubesats, the disadvantage that presents outhernet is that the reception of information is in real time according to the satellite's period, to solve this problem is proposed a satellite information reception system designed in three phases. The tracking phase consist in the antennas design knows as Double Cross and Turnstile which are used to receive information from the NOAA meteorological satellites and yagi to obtain telemetry information from the CubeSat of the OSCAR-AMSAT family, both of them are evaluated respect to the performance in different proposed scenarios. The scenarios are governed by diverse
\end{abstract}

\footnotetext{
${ }^{1}$ Escuela Superior Politécnica de Chimborazo, Facultad de Informática y Electrónica, Escuela de Ingeniería Electrónica en Telecomunicaciones y Redes, Riobamba - Ecuador, m_zabala@espoch.edu.ec

${ }^{2}$ Escuela Superior Politécnica de Chimborazo, Facultad de Informática y Electrónica, Escuela de Ingeniería Electrónica en Telecomunicaciones y Redes, Riobamba - Ecuador, garyact26@ hotmail.com

${ }^{3}$ Escuela Superior Politécnica de Chimborazo, Facultad de Informática y Electrónica, Escuela de Ingeniería Electrónica en Telecomunicaciones y Redes, Riobamba - Ecuador, mayra.pacheco@espoch.edu.ec

${ }^{4}$ Escuela Superior Politécnica de Chimborazo, Facultad de Informática y Electrónica, Escuela de Ingeniería Electrónica en Telecomunicaciones y Redes, Riobamba - Ecuador, bcasignia @ unach.edu.ec

${ }^{5}$ Escuela Superior Politécnica de Chimborazo, Facultad de Informática y Electrónica, Escuela de Ingeniería Electrónica en Telecomunicaciones y Redes, Riobamba - Ecuador, monate @espoch.edu.ec
} 
climatic factors and indoor and outdoor places around the city of Riobamba, the information received is decoded using the USB RTL-SDR dongle in conjunction with free software WxtoImg, SDRsharp, Orbitron and FOX Telemetry Analysis Tool. The second and third phase consists of the storage and distribution through a WEB server to a LAN network. The satellite images with different SNR are obtained according to each scenario, the Double Cross antenna is the most reliable in design and reception compared to the Turnstile antenna in an open environment and away from local contamination by various communication systems, with respect to the OSCAR-AMSAT satellites the yagi designed antenna works correctly in order to download telemetry information.

Keywords. technologies and sciences of engineering, Telecommunications, Satellite Communications, omnidirectional Antennas, Teledetection, Telemetry, National Oceanic and Atmospheric Administration (NOAA), CubeSat, RtlSDR (hardware)

\section{Resumen.}

La implementación de un sistema de recepción de información satelital basado en SDR parte desde el hecho de imitar el servicio de Outernet el cual es brindar información básica de meteorología, noticias, deportes, entre otras, a través de la constelación de cubesats, la desventaja que presenta outhernet es que la recepción de información en tiempo real de acuerdo al periodo del mismo, para solventar este problema se propone un sistema de recepción de información satelital diseñado en tres fases, la primera de tracking se diseña antenas Double Cross, Turnstile y yagi para evaluar el rendimiento en diferentes escenarios propuestos, las dos primeras son utilizadas a la recepción de información de los satélites meteorológicos NOAA y la tercera para la obtención de información de telemetría de los CubeSat de la familia OSCAR-AMSAT. Los escenarios propuestos se rigen a factores climáticos diversos y lugar de recepción dentro y fuera de la ciudad de Riobamba, la información recibida es decodificada utilizando el dongle USB RTL-SDR en conjunto con software libre WxtoImg, SDRsharp, Orbitron y FOX Telemetry Analisys Tool, la la segunda y tercera fase, consiste en el almacenamiento y distribucion a través de un servidor WEB a través de una red LAN. Se obtiene las imágenes satelitales con diferente SNR de acuerdo a cada escenario, 
resulta la más fiable en diseño y recepción la antena Double Cross en comparación con la antena Turnstile en un ambiente abierto y alejado de la contaminación local por diversos sistemas de comunicación, respecto a los satélites OSCAR-AMSAT la antena yagi diseñada funciona correctamente en la descarga de información de telemetría

Palabras Claves. Tecnologías y Ciencias de la Ingeniería, Telecomunicaciones, Comunicaciones Satelitales, Antenas omnidireccionales, Teledetección, Telemetría, National Oceanic and Atmospheric Administration (NOAA), CubeSat, RTL-SDR.

\section{Introducción}

Actualmente los sistemas de radiofrecuencia utilizados para el envío y transmisión de información permiten la difusión de servicios a zonas remotas con escasas de infraestructura de telecomunicaciones permitiendo así la inclusión de sectores a la era digital. Según (MINTEL, 2018) el Ministro de Telecomunicaciones y de la Sociedad de la Información, expuso que no cabe duda que la transformación digital es necesaria para encajar en la era del conocimiento, en la que vivimos. Sin embargo, a pesar de las actuales inversiones ejecutadas por los organismos internacionales en coordinación con los públicos se pretende expandir el acceso a la banda ancho móvil en el territorio nacional (Expreso, 2018). De esta manera se plantea reducir la brecha digital como una de las fases del plan nacional de telecomunicaciones presentado por el ministerio (MINTEL, 2019). El desarrollo de la industria aeroespacial permite contar con servicios de alta calidad enfocados al estudio y monitoreo del espacio utilizados en diversas áreas de investigación y enseñanza considerando las ventajas que ofrece respecto a otros sistemas de comunicación terrestres. La implementación de constelaciones de satélites en las diferentes órbitas permite ofrecer diversos servicios en tierra con sistemas de recepción especializados de alto costo. En la actualidad por la disponibilidad de hardware y software libre es posible construir el receptor para decodificar la información de ciertos satélites que transmiten en forma libre y por broadcast evitando gastos excesivos con resultados aceptables trabajos realizados. En ecuador este tipo de iniciativas se han ejecutado ya a través de proyectos de implementación de receptores multibanda de bajo costo para recepción de imágenes satelitales (Flores, G. \& 
et al. ,2018) con el objetivo de captar un rango de frecuencias de operación satelital para su posterior decodificación y utilización de información para diversos fines como lo demuestra el Observatorio Astronómico de Quito que a través de la implementación de receptores de bajo costo captan señales de los satélites NOAA para la caracterización de sitios astronómicos (Flores, G. \& et al. , 2018). Uno de los servicios gratuitos es Outernet que se crea como alternativa para la transmisión de paquetes de tamaño establecido que contiene información multimedia, radio, meteorológico, entre otras, sobre un ancho de banda limitado; para su recepción por medio de la tarjeta propia del fabricante denominado dreamcatcher (Outernet, 2018). El objetivo del proyecto es brindar una solución similar a ésta arquitectura, pero considerando la información de los satélites meteorológicos de acceso libre NOAA (NOAA, 2018) y a la información de telemetría de OSCAR-AMSAT (AMSAT EA, 2018) basado en Software Definido por Radio (SDR), también se plantea como aporte la implementación de un portal WEB para el almacenamiento y distribución a través de una red LAN, tomando así solo el concepto de Outernet para mejorarlo y ajustarlo a la información de nuestro interés.

\section{Desarrollo.}

Un satélite es un objeto artificial diseñado y construido en tierra, ubicado en órbitas específicas de acuerdo a la misión y requerimientos de funcionamiento del servicio. La principal función es transmitir información hacia el segmento terrestre para su procesamiento y almacenamiento. El conjunto de satélites es considerado una constelación con un propósito específico que de acuerdo a su ubicación y velocidad respecto a la tierra determinará su periodo de traslación, cobertura y fin. La arquitectura está conformada del segmento espacial que se compone del vehículo espacial o satélite, el segmento de control que su función es comunicarse con el satélite y controlar los parámetros de operación a través del monitoreo de mensajes de Telemetría y el control y rectificación de funcionamiento por medio de enlaces Telecomando y Control. Finalmente, el segmento de usuario engloba todas las aplicaciones en tierra (Maral G., 2009). Un CubeSat se considera a cualquier satélite que pese menos de $300 \mathrm{~kg}$ que debe cumplir con criterios específicos que controlan factores como la forma, tamaño y peso que se basan en la unidad estándar de CubeSat denominado 1U. 1U CubeSat es un cubo de $10 \mathrm{~cm}$ con una masa de aproximadamente $1 \mathrm{a} 1.33 \mathrm{~kg}$. 
Outernet es un proyecto que se basa en una red global que actualmente se encuentra en desarrollo por la organización sin fines de lucro MDIF (Media Development Investment Fund), esta organización con sede en los Estado Unidos de América se estableció en el año de 1995. Su objetivo es la proveer transmisión multimedia a receptores pequeños y portátiles de bajo costo de tasa de transmisión baja aproximada de 10 Mbytes permitiendo el acceso a información multimedia de radiodifusión, información de emergencias, educación, noticias, datos meteorológicos entre otras, sin la necesidad de registro ni tarifas recurrentes facilitando así una nueva plataforma de comunicación accesible para todos (MDIF, 2019).

RTL-SDR es un dispositivo USB fácil de usar y de muy bajo costo que recibe señales de radio RF (Radio Frecuencia). Originalmente, estos dispositivos estaban diseñados para ser utilizados como receptores DVB-T (Digital Video Broadcast-Terrestial), pero puede operar SDR genéricos, es decir solo como receptores, configurándolos en modos diferentes siendo capaces de recibir cualquier señal en el rango que generalmente es de $25 \mathrm{MHz}$ a $1.75 \mathrm{GHz}$ (Stewart B. \& et al., 2015) las cuales son convertidas en banda base, las digitaliza y el dispositivo envía muestras de la señal de banda base a través de su interfaz USB hacia el software de procesamiento digital seleccionado.

\section{Metodología}

Se plantea un sistema de recepción de imágenes satelitales e información de telemetría de los satélites NOAA y OSCAR-AMSAT, se construye antenas basado en los parámetros de transmisión. Para la demodulación de la información se utiliza el SDR en conjunto con software SDRsharp (RTL-SDR, 2019) y WxtoImg (WmtoImg, 2019) para visualización de las imágenes. En el caso del satélite OSCAR-AMSAT será necesario el software FOX1 Telemetry Analys Tool disponible en (Amateur Radio in Space, 2019).

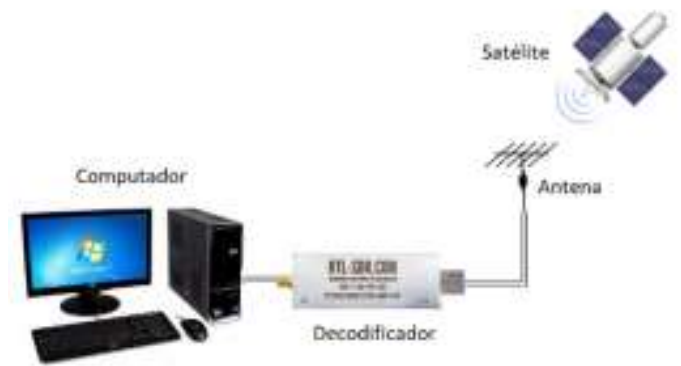

Figura 1. Esquema de implementacion del sistema de recepción de información satelital 


\section{Antenas}

- Yagui

Para la recepción de la telemetría de la familia de los satélites OSCAR-AMSAT el AO-85, AO-91 y AO-92 se utiliza una antena yagi con frecuencia de operación de 437 MHz en la banda UHF, el diseño de la antena consta de 5 directores, 1 reflector adicional y un dipolo. Para encontrar la longitud de cada elemento y la separación a detalle en (Cabezas G., 2018).

- Turnstile

Esta es una de las antenas más simples y económicas que se puede realzar para la recepción de las imágenes en formato APT (Automatic Picture Transmision), presenta un diagrama de radiación omnidireccional (Cabezas G., 2018), lo que beneficia en la captación de la señal desde el satélite, a diferencia de las antenas directivas.

\section{- Double Cross}

La antena Double Cross posee un diagrama de radiación que presenta un área de cobertura de media esfera el cual permite que la recepción de la señal de los satélites sea mucho mejor. Para el correcto funcionamiento de esta antena se debe de considerar dos parámetros fundamentales los cuales son la frecuencia de trabajo y la polarización (Cabezas G., 2018).

Sistema de recepción de información satelital

El sistema se plantea en 3 fases (Ver Figura. 2):

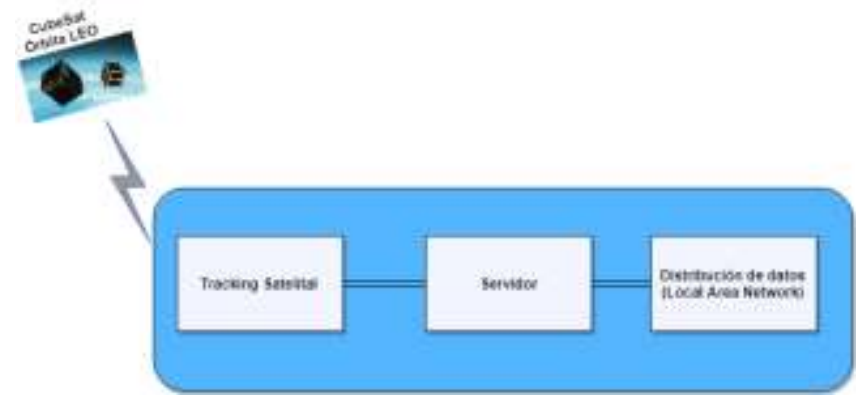

Figura 2. Sistema de Recepción de información satelital 
- Fase I: Tracking Satelital

En la Figura. 3 se observa la primera fase de la implementación la cual es el tracking satelital el cual consta del diseño y construcción de antenas, configuración del RTL-SDR y decodificación de imágenes satelitales.

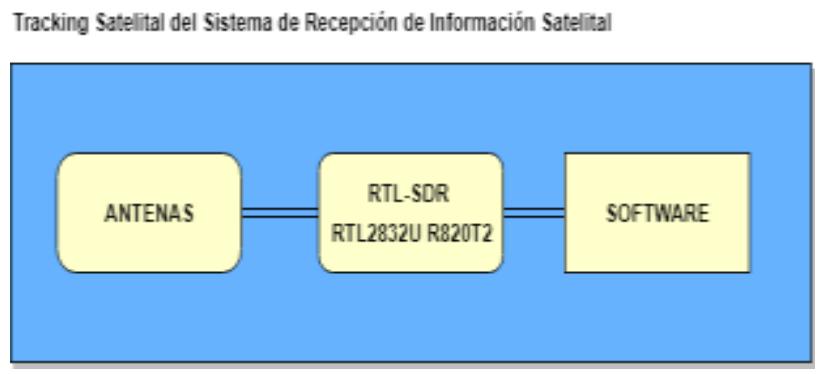

Figura 3. Subsistema de Tracking

- Fase II: Servidor

El aporte de la propuesta es el almacenamiento de la información decodificada en un sistema de gestión base de datos para la creación sitio web de publicación. La implementación esta esquematizado en Figura. 4, se realiza en WAMP-SERVER, para mayor detalle de la implementación verificar en (RADIO DIGITAL URE, 2016).

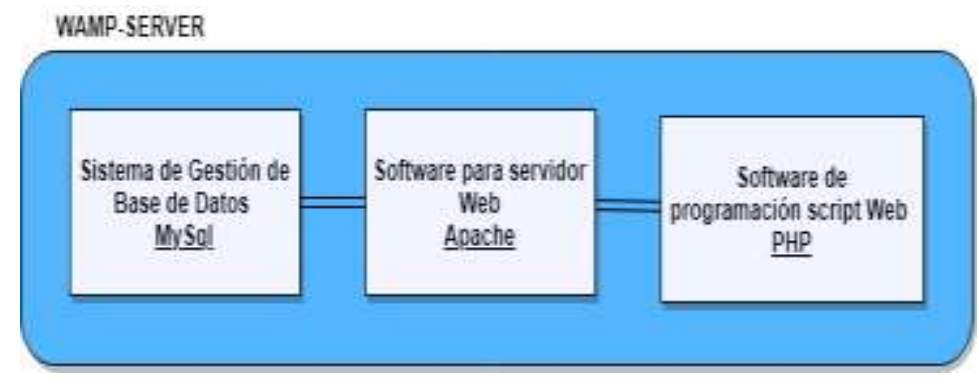

Figura 4. Subsistema de almacenamiento web

- Fase III: Distribución de datos

Una vez implementado el servidor es posible la distribución y acceso a la información satelital decodificada por medio una interfaz de interacción con el usuario o portal web (Ver Figura. 5) para descargar la información de interés. 


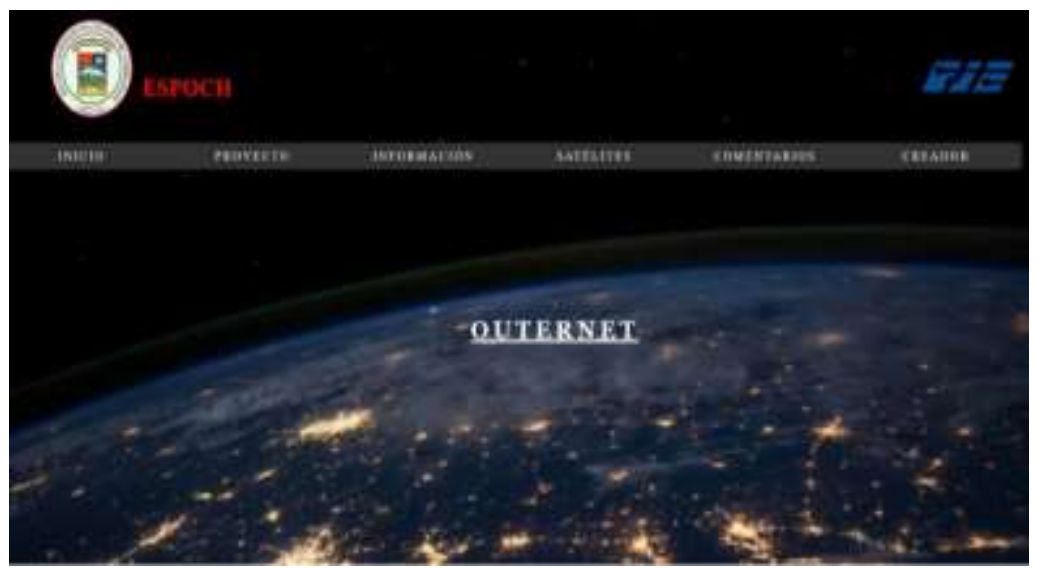

Figura 5. Interfaz web

\section{Resultados}

Se propone escenarios de prueba de diferentes condiciones bajo el cual se pone a prueba el rendimiento de las antenas construidas y el nivel de SNR en el receptor y así determinar cuáles de ellas son las mejores condiciones para la recepción de la señal.

- Escenario 1.- Antena Double Cross en Ausencia de lluvias.

En la Figura. 6 se muestra la imagen del día 28 de mayo del 2018 tomada a las 18:03 horas en ausencia de lluvias y cielo despejado, la imagen muestra ruido después de 10 minutos de la recepción.

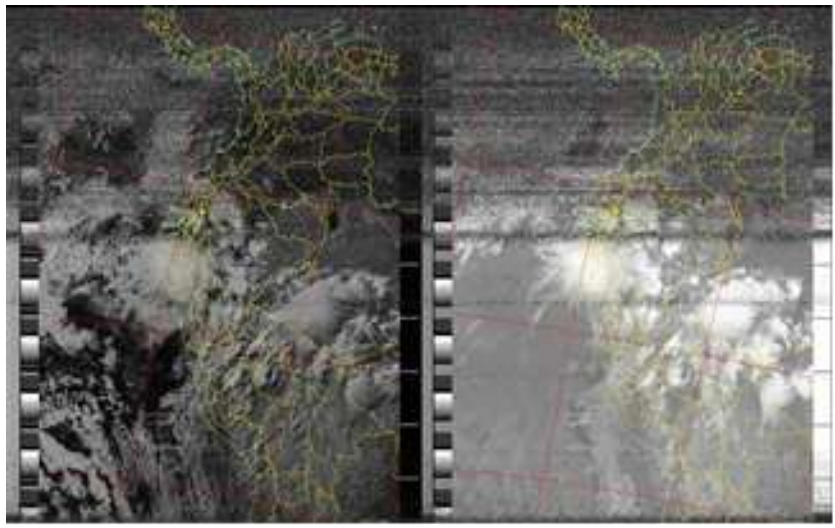

Figura 6. NOAA 15, 28 de mayo-2018 - Antena Double Cross - Ausencia de lluvia

El software WxtoImg permite aplicar filtros de color, la herramienta es Multi Spectral Analysis (MSA) con esto se consigue mejorar la calidad de las imágenes asignando coloración a la nubosidad, tierra y mar como se observa en la Figura 7. 


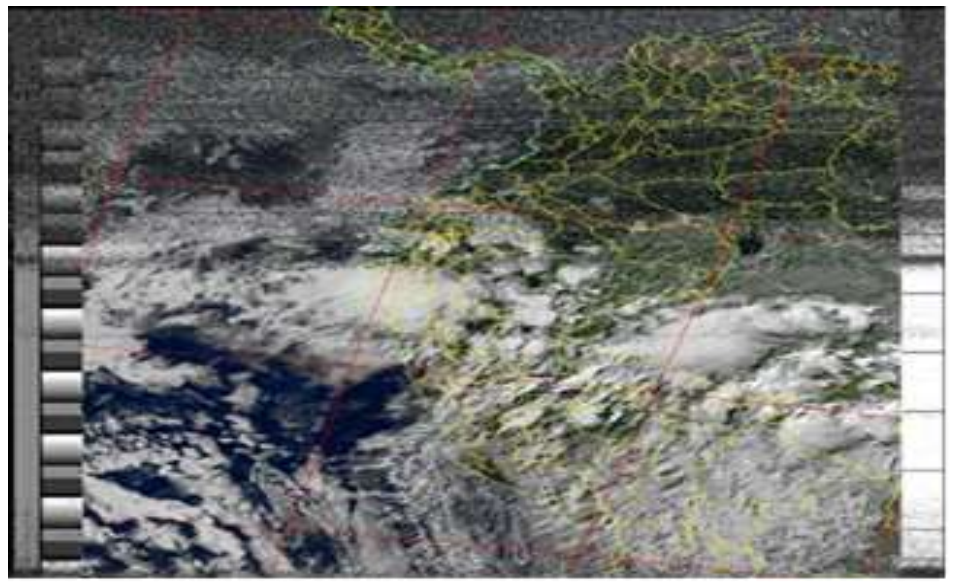

Figura 7. Imagen con MSA de NOAA 15 del 28 de mayo 2018

Bajo estas condiciones de cielo despejado y en ausencia de lluvia se obtuvo una imagen legible, con un SNR de $26 \mathrm{~dB}$ como se observa en el análisis de espectro en la Figura 8. Los niveles de señal teóricos para la recepción de una buena señal son entre 10 - 31,024 dB por lo tanto con un nivel de $26 \mathrm{~dB}$ la imagen es considerablemente buena.
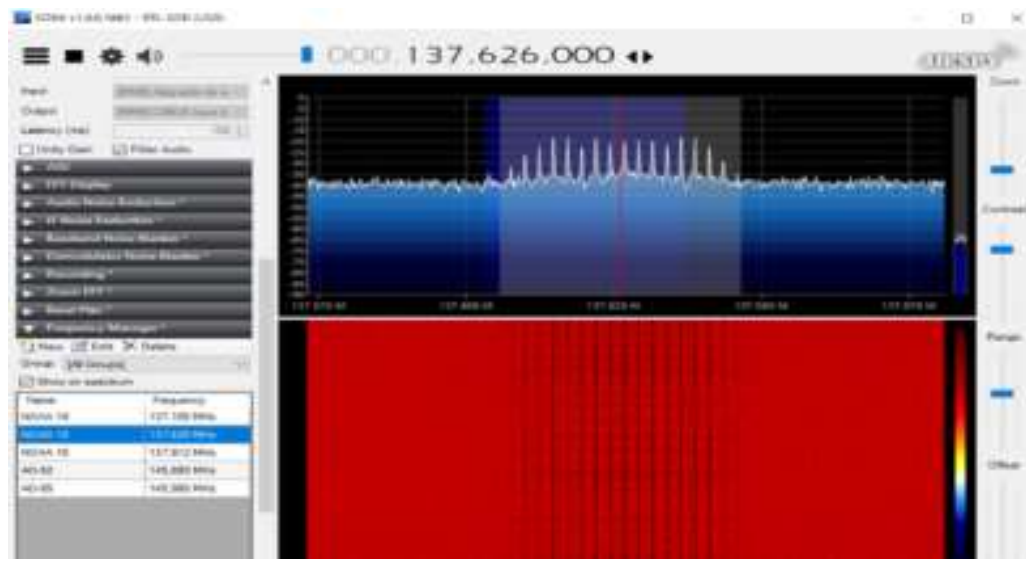

Figura 8. Análisis de espectro NOAA 15

- Escenario 2.- Antena Double Cross en presencia de Fuertes Lluvias

En la Figura 9 capturada el día 23 de mayo del 2018 a las 17:30 horas presenta ruido en exceso debido a la presencia de lluvias y viento fuerte durante la recepción provocando la inestabilidad de la antena, se puede observar que, debido a la lluvia, la imagen muestra densa concentración de nubes. 


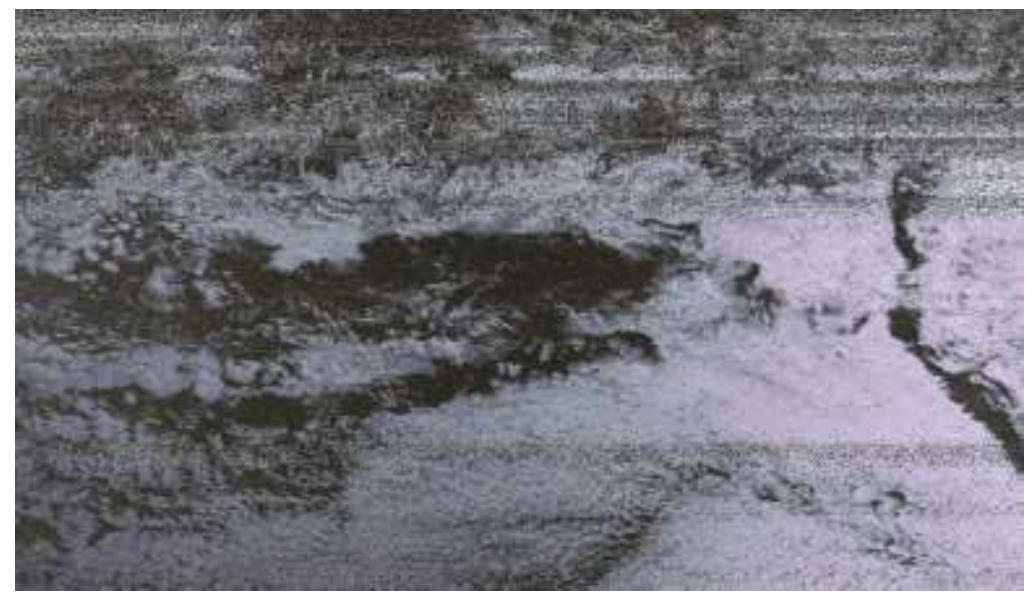

Figura 9. NOAA 18, 23 mayo 2018- Antena Double Cross - Lluvia Fuerte

En la Figura 10 se observa el análisis del espectro de la señal recibida bajo estas condiciones climáticas y se puede notar que el SNR es de $10 \mathrm{~dB}$ el cual es inferior al del escenario 1 y este nivel de SNR se encuentra en el margen inferior del nivel teórico analizado anteriormente por lo que se justifica la mala calidad de la imagen.

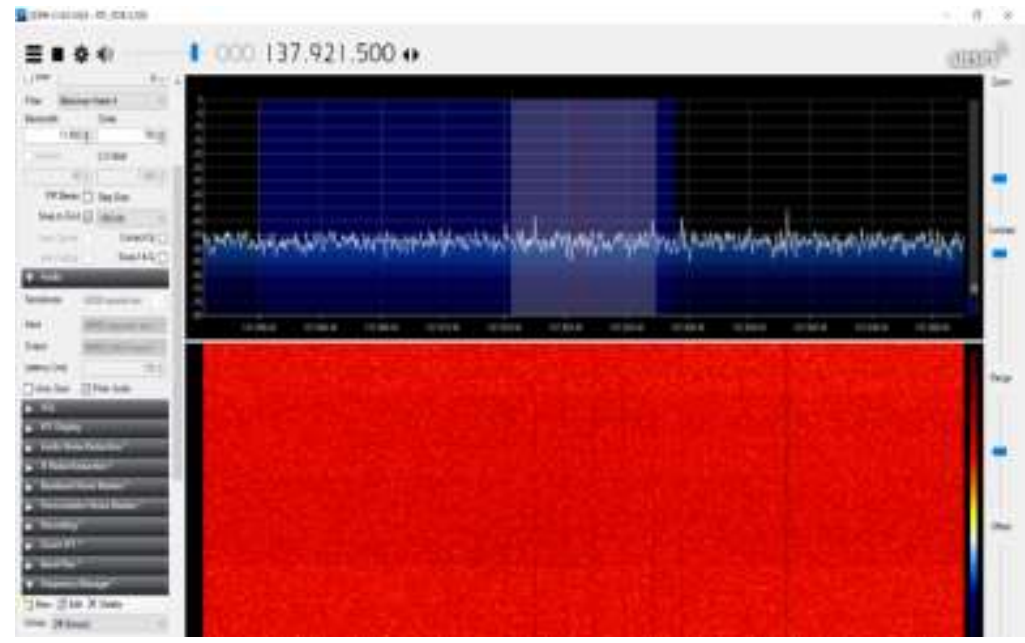

Figura 10. Análisis de espectro NOAA 18 presencia de lluvias

- Escenario 3.- Antena Turnstile en Ausencia de lluvias

La principal desventaja de la antena Turnstile es la escasa ganancia, en la Figura 11 se muestra que la antena no es capaz de capturar una imagen amplia sin ruido, sólo capta la 
señal del satélite por alrededor de 2 o 3 minutos pasado ese tiempo solo captura interferencias y ruido.

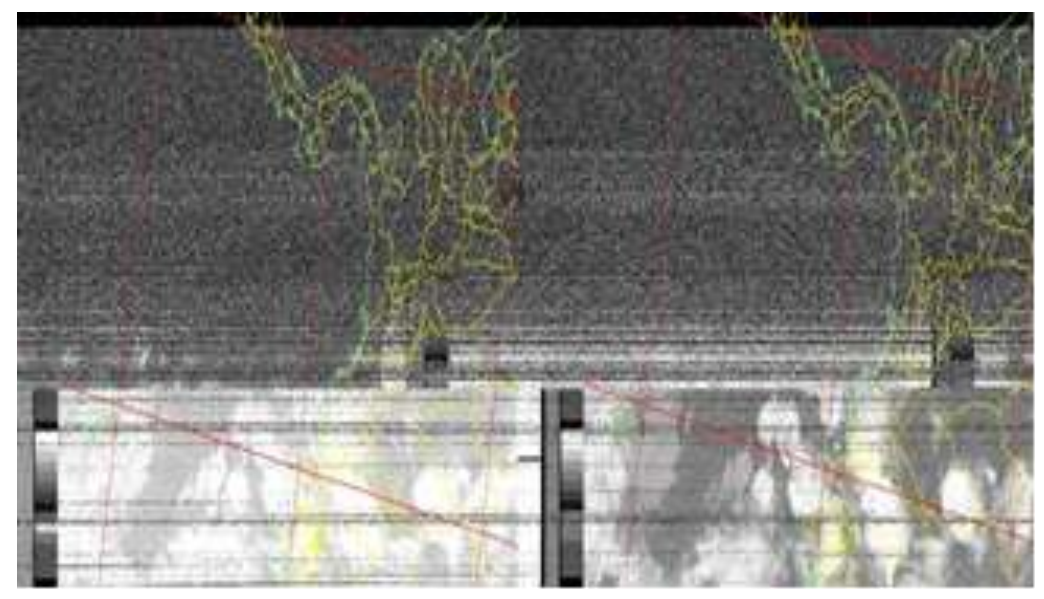

Figura 11. Imagen con antena Turnstile en ausencia de lluvia

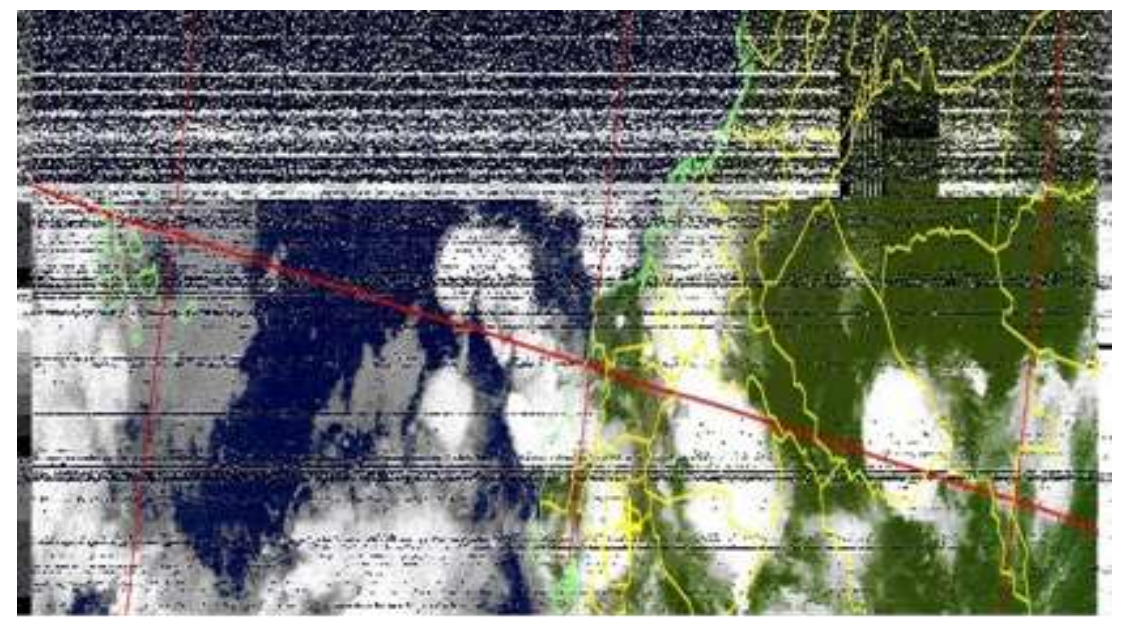

Figura 12. Imagen Filtro MSA antena Turnstile en ausencia de lluvia

El análisis del espectro de la imagen durante los 3 minutos de recepción se muestra en la Figura. 13 el cual indica un nivel de SNR de $26 \mathrm{~dB}$ igual al que la antena Double Cross a diferencia de que la recepción de esta última dura más tiempo por lo que la imagen es mucho más amplia y con menos ruido. De igual manera este nivel de señal es muy bueno y se encuentra dentro del rango teórico. 


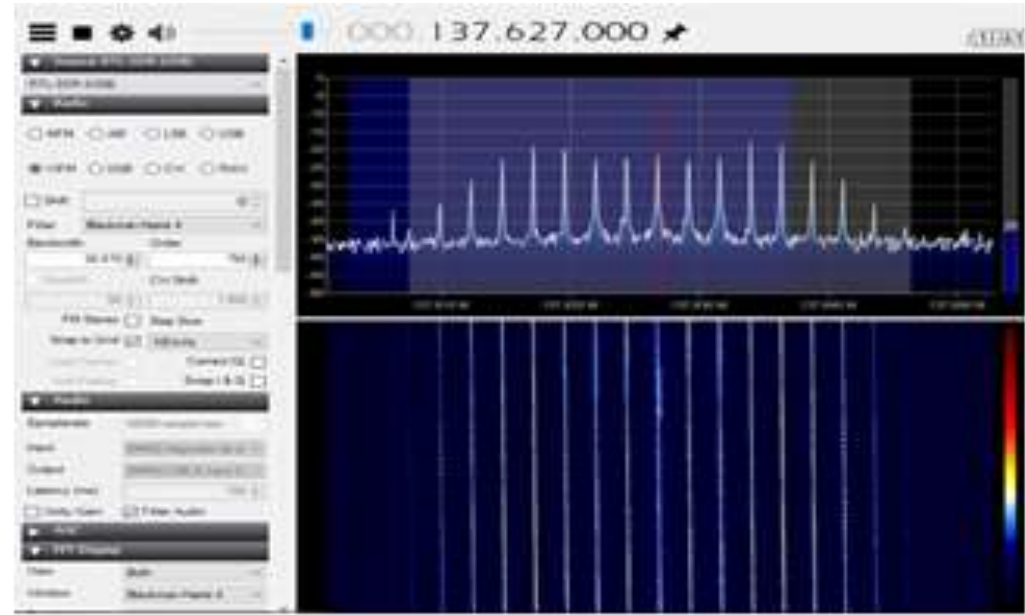

Figura 13. Análisis de espectro NOAA 15 antena Turstile en ausencia de lluvias

- Escenario 4.- Antena Turnstile con Fuertes Lluvias.

En la Figura. 14 se observa la presencia de ruido en la imagen la antena Turnstile tiene problemas con respecto a la ganancia y solo es capaz de recibir las señales satelitales por no más de 3 minutos.

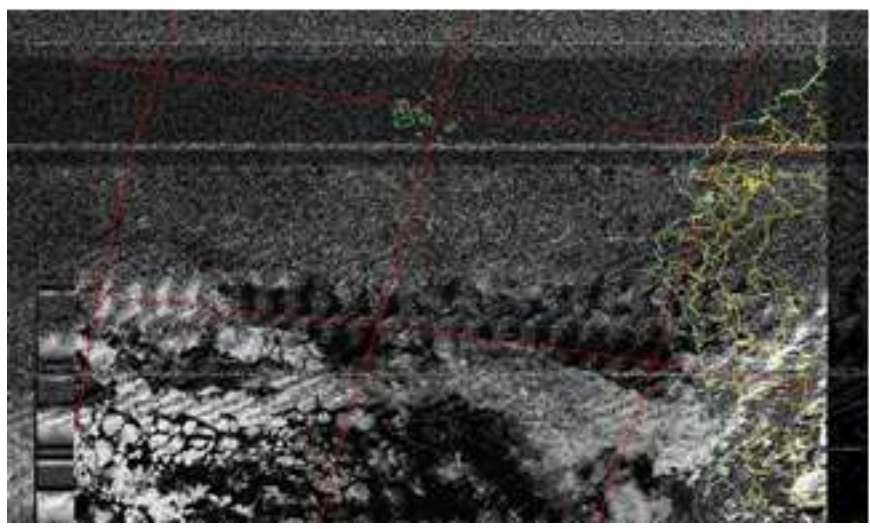

Figura 14. NOAA 19 con Filtros antena Turnstile con fuertes lluvias

En cuanto al análisis del espectro bajo estas condiciones, se observa que la relación señal a ruido es de solo $10 \mathrm{~dB}$ muy inferior al escenario anterior de la misma antena, con este nivel de SNR no es posible obtener una buena imagen porque se encuentra en el límite teórico inferior calculado anteriormente, por estas razones se determina que esta antena no funciona correctamente bajo estas condiciones climáticas. 


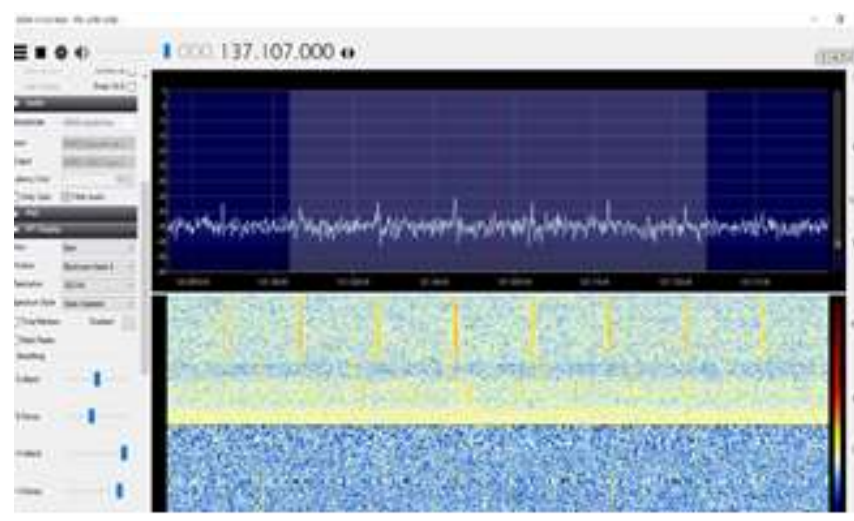

Figura 15. Análisis de espectro NOAA 19 antena Turnstile con lluvia fuerte

- Escenario 5: Fuera de la ciudad.

La recepción de la imagen tomada el 3 de junio del 2018 a las 16:17 horas de Ecuador en el refugio del Volcan Chimborazo, bajo buenas condiciones climáticas, cielo despejados y ausencia de lluvias además considerando un factor importante el cual es la lejanía a otras posibles interferencias. Las pruebas se hicieron con la antena Double Cross ya que en experimentos anteriores esta demostró un mejor rendimiento

En las Figura 16 - 17 se puede observar que la imagen mejoro considerablemente, existe menos ruido y se recibe imágenes de mayor cobertura cubriendo territorio colombiano, venezolano y brasileño.

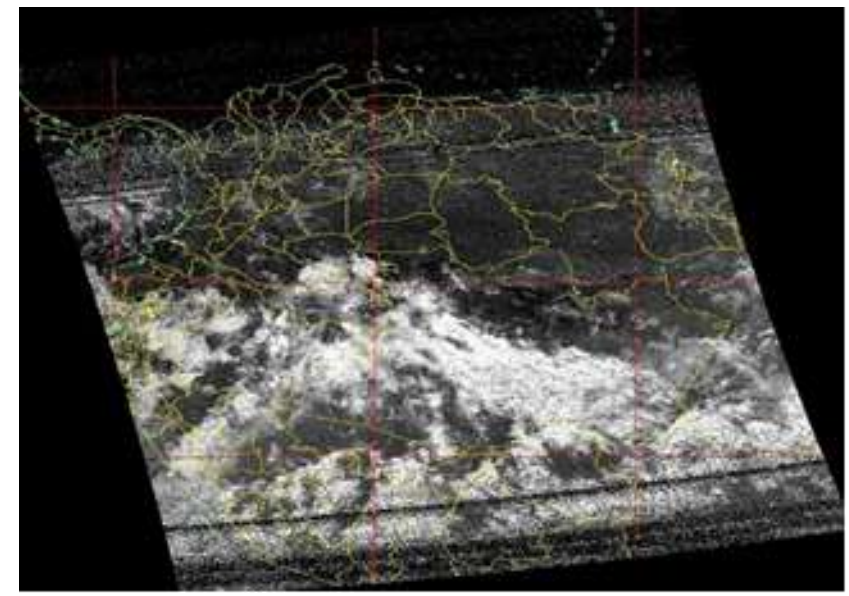

Figura 16. Imagen Antena Double Cross fuera de la ciudad 


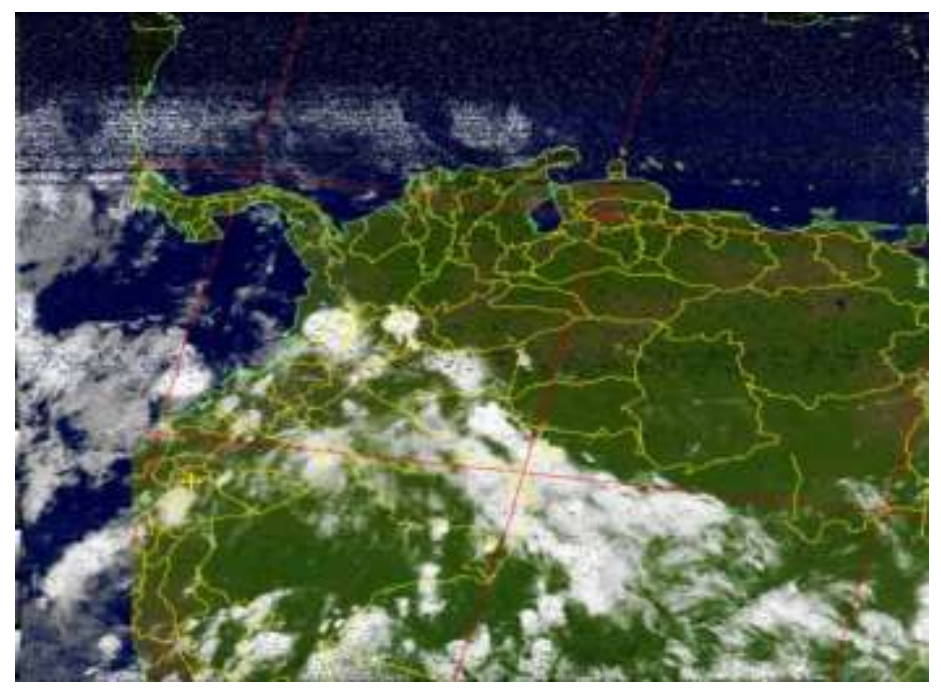

Figura 17. Imagen con filtros MSA antena Double Cross fuera de la ciudad

Además, en la Figura 16 se observa el análisis de espectro con un SNR de 29 dB el cual es el mejor SNR obtenido con los satélites NOAA y además es el que más se acerca al límite superior de $31,024 \mathrm{~dB}$ calculado.

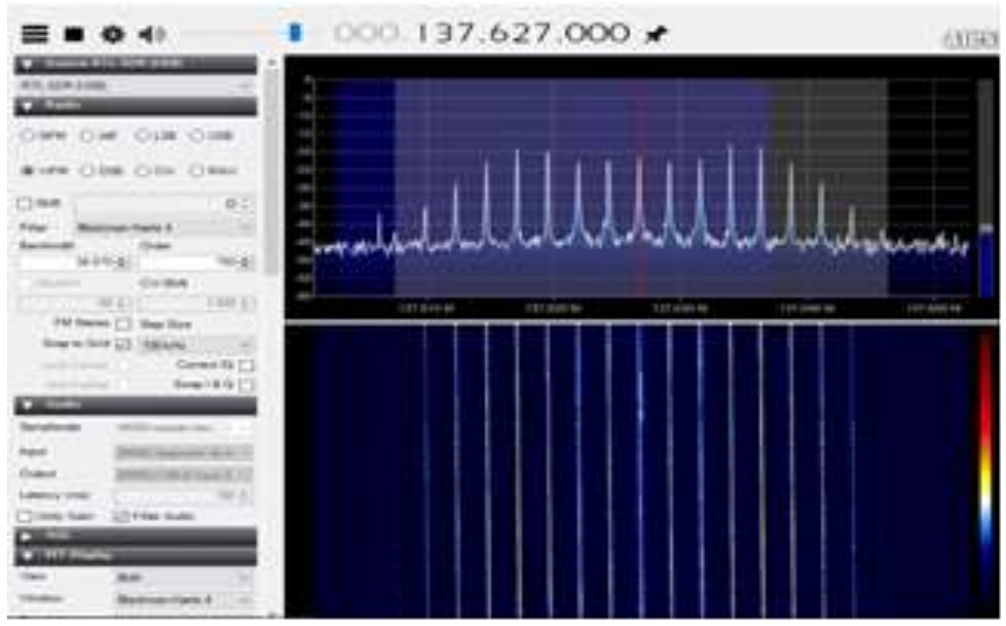

Figura 18. Espectro antena Double Cross Fuera de la ciudad

\section{Conclusiones.}

- Para la adquisición de señales de los satélites NOAA y OSCAR-AMSAT es necesario la construcción de una estación terrena que consta de antenas diseñadas de acuerdo a 
las características de operación del sistema de comunicación satelital, así como de un decodificador de la señal que se realiza a través de la tecnología SDR para el posterior procesamiento a través de software libre en el ordenador.

- Los niveles de señal en el receptor teóricos calculados dan un valor aproximado de SNR mínimo de $10 \mathrm{~dB}$ y máximo de $31.024 \mathrm{~dB}$ lo que nos da una referencia para las pruebas establecidas, las cuales se proponen 6 escenarios en diversas condiciones climáticas para evaluar el rendimiento y versatilidad de las antenas. Para la recepción de las imágenes satelitales del satélite NOAA se evalúa la antena Double Cross y Turnstile comparando su rendimiento en la recepción de la señal, en ambos casos se obtienen valores similares de $26 \mathrm{~dB}$ en ausencia de lluvias y $10 \mathrm{~dB}$ con fuertes lluvias, sin embargo la antena Double Cross muestra mayor tiempo de conexión con el satélite en un intervalo de 5-6 minutos mostrando una cobertura mayor a diferencia de la antena Turnstile que mantuvo un tiempo de conexión de 4 minutos esto se debe principalmente a la ganancia que presenta, lo cual la hace una antena con poca confidencialidad en recepciones de imágenes en formato APT.

- En lugares altos, despejados y poco saturados por los demás sistemas de comunicación inalámbricos, la antena Double Cross presenta mejor rendimiento obteniendo una SNR de $29 \mathrm{~dB}$ siendo este el mejor obtenido respecto a los demás escenarios, como es el caso del escenario en las faldas del volcán Chimborazo ubicado aproximadamente a $4386 \mathrm{msnm}$,

- El servicio de Outernet es propiedad de la empresa estadounidense del mismo nombre con infraestructura propia de software y hardware para la recepción de información satelital de su constelación, sin embargo, por la limitación del acceso a estos recursos se opta por utilizar RTL-SDR y software libre como alternativa para la creación de una estación terrena propia proponiendo un servicio similar al de Outernet. El dispositivo RTL-SDR sirve para la recepción de las imágenes satelitales sus características son óptimas para este tipo de trabajos y además reducen drásticamente la infraestructura del hardware que hoy en día son utilizadas en la meteorología, adicional a esto su precio es accesible, así que para radioaficionados que se estén iniciando en este tema este dispositivo es una buena herramienta, pero hay que tener en cuenta restricciones de uso. 


\section{Referencias bibliográficas.}

[1] Ministerio de Telecomunicaciones y de la Sociedad de la Información. (2018). "MINTEL promueve el desarrollo del país, a traves de la reduccion de la brecha digital y la apropiacion de las TIC". Quito-Ecuador: https://www.telecomunicaciones.gob.ec/mintel-promueve-el-desarrollo-del-pais-atraves-de-la-reduccion-de-la-brecha-digital-y-la-apropiacion-de-las-tic/

[2] Expreso. (2018), "Invierten 50 millones de dólares para reducir la brecha digita en Ecuador". Guayaquil-Ecuador: https://www.expreso.ec/actualidad/inversion-bid-brechadigital-ecuador-EX2462226

[3] Ministerio de Telecomunicaciones y de la Sociedad de la Información. (2019). "Plan Nacional de Telecomunicacioners y Tecnologias de Informacion del Ecuador 20162021”. Quito-Ecuador: https://www.telecomunicaciones.gob.ec/wpcontent/uploads/2016/08/Plan-de-Telecomunicaciones-y-TI..pdf

[4] Flores, G. \& et al. (2018). Receptor Multibanda de Bajo Costo para la Recepción de Imágenes de Satélites Meteorológicos y SSTV. Rev Politéc. 2018, vol.40, n.2, pp.25-30. ISSN 2477-8990.

[5] Flores, G. \& et al. (2018). Low Cost NOAA Satellite Signal receiver for Characterization of Astronomical Sites. Astronomical Observatory of national Polytechnic School, Quito Ecuador.

[6] Outernet, (2018). Outhernet. https://Outernet.is/

[7] NOAA, (2018).Satélites Meteorologicos. https://www.noaa.gov/

[8] AMSAT EA. (2018). AMSAT EA - Satélites OSCAR. https://www.amsat-ea.org/lossatélites-oscar/.

[9] Maral G., Bousquet M. \& Sun Z., (2009), Satellite Communications Systems: Systems, Techniques and Technology,", 5th ed., John Willey.

[10] MDIF (2019). Media Development investment Fund. https://www.mdif.org/invest-inmedia-development/

[11] Stewart B., Barlee K., Atkinson D., \& Crockett .L, (2015). Software Defined Radio using Matlab \& Simulink and the RTL-SDR, Galsgow, Scotland, UK, $1^{\text {st. }}$. edition, Department of Electronic and Electrical Engineering, University of Strathyde 
[12] RTL-SDR. (2019). AirSpy.https://airspy.com/download/

[13] WmtoImg. (2019). WmtoImg Restored software to decode APT and WEFAX signals from weather satellites, https://wxtoimgrestored.xyz/

[14] Amateur Radio in Space, (2019). FoxTelem Software for Windows, FOX Telemetry Decoder. Disponible en: https://www.amsat.org/foxtelem-software-for-windows-maclinux/

[15] Cabezas G., (2018). Análisis e Implementación del Servicio de Outernet por Medio de Micro Satélite para la recepción de Información en el Laboratorio de Microondas y Comunicaciones de la Facultad de Informática y Electrónica, Tesis pregrado, Escuela de Ingeniería en Telecomunicaciones y Redes, ESPOCH, Riobamba, Ecuador.

[16] Cabezas G., (2018), Análisis e Implementación del Servicio de Outernet por Medio de Micro Satélite para la recepción de Información en el Laboratorio de Microondas y Comunicaciones de la Facultad de Informática y Electrónica”, Tesis pregrado, pp. 44 46, Escuela de Ingeniería en Telecomunicaciones y Redes, ESPOCH, Riobamba, Ecuador.

[17] Cabezas G., (2018), Análisis e Implementación del Servicio de Outernet por Medio de Micro Satélite para la recepción de Información en el Laboratorio de Microondas y Comunicaciones de la Facultad de Informática y Electrónica, Tesis pregrado, pp. 47 - 51, Escuela de Ingeniería en Telecomunicaciones y Redes, ESPOCH, Riobamba, Ecuador.

[18] RADIO DIGITAL URE. (2016). Foro de la Vocalìa de Radio Digital. https://radiodigital.ure.es/foro/viewtopic.php?t=333.

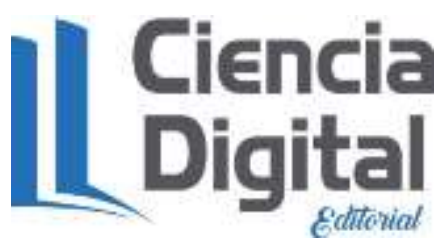




\section{PARA CITAR EL ARTÍCULO INDEXADO.}

Zabala, M., Cabeza, G., Pacheco, M., Casignia, B., \& Oñate, A. (2019). Sistema de recepción de información satelital basado en SDR. Ciencia Digital, 3(2.6), 446-463. https://doi.org/10.33262/cienciadigital.v3i2.6.591

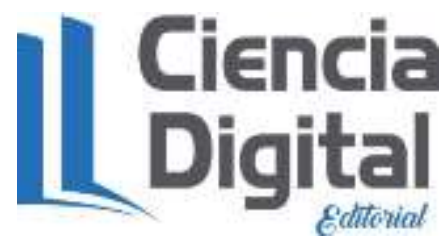

El artículo que se publica es de exclusiva responsabilidad de los autores y no necesariamente reflejan el pensamiento de la Revista Ciencia Digital.

El artículo queda en propiedad de la revista y, por tanto, su publicación parcial y/o total en otro medio tiene que ser autorizado por el director de la Revista Ciencia Digital.
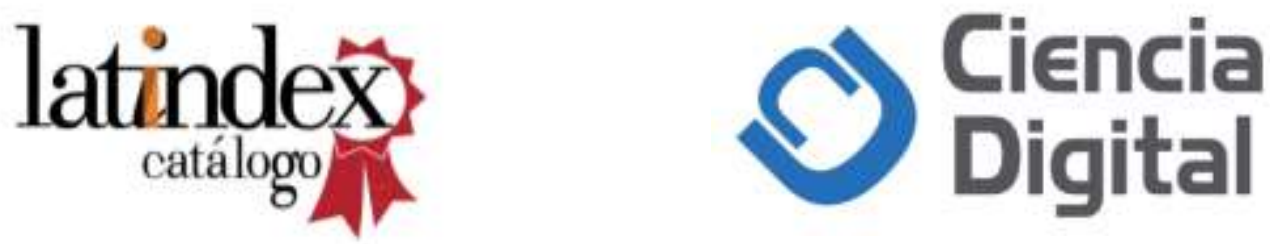